\title{
Predicción de precios del petróleo y el aluminio usando ecuaciones diferenciales estocásticas
}

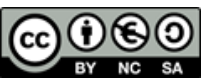

\section{Prediction of oil and aluminum prices using stochastic differential equations}

Flavia Panelas Ramos. ${ }^{1}$, Camilo Rivera Rodríguez. ${ }^{2} \&$ Karen Santos Fernández. ${ }^{3}$

Recibido: 05-09-2018 / Revisado: 13-09-2018 / Aceptado: 21-09-2018 / Publicado: 05-10-2018

Resumen. $\quad$ DOI: $\underline{\text { https://doi.org/10.33262/concienciadigital.v1i4.906 }}$

En el mundo globalizado de hoy, la incertidumbre se muestra en el desempeño de las economías modernas. Esta condición influye directamente en estas economías, por tanto, la propensión a sufrir las consecuencias de las fluctuaciones de las diferentes variables de mercado es un hecho incuestionable. La apertura comercial y financiera que distingue a las economías modernas en un contexto global ha potenciado la aparición de escenarios inciertos donde el riesgo juega un papel protagónico. Esta situación requiere de la implementación de programas para administrar riesgos con vistas a reducir los impactos de los conflictos financieros que atentan contra la estabilidad de los diferentes actores económicos.

En general, las economías pequeñas y abiertas presentan características específicas que las hacen muy vulnerables frente a los desequilibrios en las relaciones económicofinancieras, entre ellas, las variaciones de los precios. Por lo tanto, gestionar la exposición a la volatilidad de los precios genera una creciente necesidad de protección financiera.

Cuba se inserta en este tipo de economías altamente expuestas a las variaciones dadas en el entorno internacional. Dicha vulnerabilidad se ve profundizada con la existencia del embargo económico y financiero impuesto por los Estados Unidos. Debido a esta situación, resulta beneficioso para la Isla contar con herramientas para reducir al

\footnotetext{
${ }^{1}$ Universidad de la Habana, La Habana, Cuba, fpenelas@gmail.com.

${ }^{2}$ Universidad de la Habana, La Habana, Cuba, crivera@gmail.com.

${ }^{3}$ Universidad de la Habana, La Habana, Cuba, ksantos@gmail.com.
} 
mínimo los riesgos y, optimizar el proceso de toma de decisiones en las cuestiones relacionadas con el comercio exterior.

Palabras claves: petróleo, aluminio, ecuaciones diferenciales estocásticas.

\section{Abstract.}

In the globalized today's world, uncertainty shows up in the modern's economies performance. This condition influences directly on these economies, because of that, the propensity to suffer the market's different variables' fluctuations' consequences is an unquestionable fact. The commercial and financial opening that distinguish the modern economies on a global context has encouraged the appearance of uncertain sceneries where the risk plays a starring role. This situation requires de implementation of programs to administrate risk with financial conflict's impact reduction views that attempt against the different economic authors' stability.

Generally, small and open economies present specific characteristics that makes them very vulnerable against economic financials unbalances, amongst them, price variation. That is why; to manage the exposure to prices volatility generates a growing need for financial protection.

Cuba enters in this type of economy highly exposed to the given variations in the international scenery. This vulnerability goes deeper with the existence of the economic and financial embargo imposed by the United States. Due to this situation, it is highly beneficious to the island to count with tools to reduce to the minimum risk, and optimize the making decisions's process on the external commerce's related questions.

Keywords: oil, aluminum, stochastic differential equations.

\section{Introducción.}

Como se conoce nada es estático en la Economía desde las propias tendencias económicas y escuelas hasta los procesos económicos en sí. Conocer cómo se comportarán diferentes fenómenos a lo largo del tiempo resulta tan imprescindible como complejo.

Fluctuaciones y pronósticos de precios de materias primas vitales. ¿Tendrá ello algún tipo de interés para economistas, ministerios, y en general "hacedores de políticas"? Indudablemente conocer cómo se comporta y comportará uno de los componentes más esenciales para la reproducción de los capitales, los insumos, es de importancia vital para el desarrollo de un país, para la aplicación y proyección de las más eficientes políticas. Cómo se rige un bien en el mercado, y así poder trazar estrategias para lograr adelantarse a los altibajos del mercado, haciendo posible el trazo de políticas que los compensen o aprovechen. 
La Economía y la Matemática sin dudas van de la mano en todo momento. Todo fenómeno económico hasta el más subjetivo puede de una manera u otra ser llevado a un modelo. Dadas estas prerrogativas la herramienta por excelencia es la Ecuación diferencial. Las ecuaciones diferenciales modelan los fenómenos dinámicos, de ahí su aplicación en economía y finanzas. Cómo si no lograr un modelo que tenga presente las tasas de cambio de varios parámetros y actualizándolo con las pertinentes constantes relativas al momento específico encontrar una curva que describa eficientemente la incógnita o proyección en cuestión. Fue posible encontrar diferentes aristas dentro de la economía donde se utiliza esta herramienta. Además, gracias a los conocimientos adquiridos principalmente durante el semestre y la investigación conjunta del equipo se logró modelar los precios del petróleo y el aluminio, con el objetivo de lograr pronósticos acertados y puede que incluso promover el uso de esta herramienta matemática más compleja a nivel nacional.

\section{Objetivos:}

- Explicar la teoría y aplicaciones de las ecuaciones diferenciales ordinarias.

- Introducir las ecuaciones diferenciales estocásticas y los modelos de reversión a la media.

- Modelar los precios diarios del aluminio y el petróleo.

- Verificar el ajuste de los modelos.

- Predecir un comportamiento aproximado un año después de la toma de datos.

- Proponer la difusión de esta herramienta para aplicación en cuestiones económicas o sociológicas de vital interés para el país.

\section{Desarrollo:}

\section{Modelo matemático}

Con frecuencia se desea describir el comportamiento de algún sistema o fenómeno de la vida real en términos matemáticos; dicho sistema puede ser físico, sociológico o hasta económico. La descripción matemática de un sistema o fenómeno se llama modelo matemático y se forma con ciertos objetivos en mente; por ejemplo, predecir el comportamiento de cierta variable aleatoria.

Un modelo matemático puede ser descrito mediante una ecuación diferencial o un sistema de ellas, basados en información previa del investigador. Luego se deben estimar los parámetros del modelo y revisar su ajuste.

\section{Administración de riesgos en commodities.}

La situación actual del mercado se muestra cada vez más compleja, en tanto la globalización incide de manera determinante y aún más evidente en el funcionamiento del mismo. En este sentido, los niveles de interconexión e interdependencia provocan el desarrollo de un mercado único donde los riesgos están cada vez más representados. Ello conlleva, por un 
lado, a un incremento en la vulnerabilidad de las empresas en un contexto que sufre cambios constantemente, y por otro, el movimiento de capitales se facilita y afecta la economía de un país si no son controlados correctamente.

Asociado a ello, la gestión de riesgos empresariales se percibe hoy como un tema de importancia relevante. El enfoque moderno de la administración de riesgos, es decir, la administración integrada de riesgos constituye un proceso que, de forma integral, sistemática y disciplinada, le permite a la organización evaluar, controlar, explotar, financiar y supervisar sus riesgos significativos con la finalidad de incrementar sus beneficios en el corto y largo plazo. Su correcta aplicación:

- Favorece el aumento de la comprensión total de riesgos.

- Facilita, a la alta dirección, una representación del nivel de afectación posible.

- Desarrolla un proceso sistemático, cooperativo y de organización para que la compañía utilice la identificación, atención y el financiamiento o contingencia para el riesgo.

- Posibilita concentrarse en las decisiones de la administración del riesgo que maximizan las ganancias, tomando al riesgo como una oportunidad para generar ingresos.

- Mejora la capacidad de la organización para identificar los eventos potenciales y establecer respuestas.

- Eleva la efectividad en la administración del tiempo y de los recursos del negocio para manejar riesgos, proporciona una mayor capacidad para identificar y evaluar tales riesgos y establecer niveles aceptables del mismo en relación con el potencial de crecimiento y el logro de objetivos: racionaliza el capital.

Por lo general, el mercado que más incertidumbre genera debido a la alta volatilidad de los precios de sus productos es, precisamente, el mercado de commodities. En este sentido, resulta interesante analizar sus características, en función de identificar los instrumentos a utilizar a fin de cubrirse del riesgo explicado por el movimiento de los precios.

\section{Análisis fundamental}

El análisis fundamental es uno de los enfoques más utilizados por los analistas para estudiar los mercados financieros y tratar de predecir los movimientos de los precios pues se centra en las causas de dichas variaciones. Este abarca un gran número de variables, dependiendo de la naturaleza del activo y el mercado en el que se negocia, y se debe desarrollar continuamente para que pueda ser efectivo.

En el análisis fundamental se internalizan elementos de diversas ramas, entre ellas, la microeconomía, la contabilidad y la macroeconomía. En este sentido, se distinguen tres fases por las que transita esta interpretación cualitativa. Por un lado, se detiene en el análisis 
político y macroeconómico donde se tienen en cuenta aspectos relacionados con la situación política, financiera, coyuntural y social del país, los precios (inflación); así como su credibilidad externa (riesgo país, tipo de cambio). Por otro lado, se desarrolla el análisis del sector industrial que se refiere a la relación oferta-demanda del activo subyacente en cuestión (niveles de producción, reservas, entre otros). Por último, se llega al nivel empresarial donde se analiza la posición de la empresa, su contabilidad e informes financieros, de forma que se conozca su nivel de endeudamiento, solvencia, liquidez, entre otras ratios de interés.

De acuerdo con el análisis que se desee desarrollar, este método se puede dividir en dos variantes que no son excluyentes entre sí, sino que lo recomendable sería extraer las conclusiones de ambos análisis y combinarlas para hacer un estudio más completo e integral del fenómeno.

Análisis Top-Down: Es el análisis que describe una trayectoria descendiente, es decir de arriba hacia abajo, de lo general y global a lo concreto y particular. Atraviesa por tres niveles fundamentales: la economía en su conjunto, posteriormente se hace un análisis sectorial y en último lugar se analiza a la empresa en sí.

Análisis bottom-up: Se hace el análisis en sentido inverso, es decir se parte de lo concreto, pero ni la coyuntura a nivel sectorial ni la economía nacional son importantes.

La relación oferta-demanda es el único factor a considerar en la sencilla teoría del análisis fundamental, que se puede sintetizar en un solo principio: Menores existencias o un mayor consumo hacen que se eleven los precios y la abundancia de suministros o una reducción del consumo provocan una reducción de los mismos.

En este sentido, es importante hacer alusión a algunos factores que influyen en el análisis fundamental como: las reservas, la producción, la posición geográfica, las condiciones climatológicas, las condiciones económicas, la inflación, las expectativas, las imperfecciones del mercado, los factores competitivos y políticos, las variaciones en el tipo de cambio, los ratios e índices, entre otros.

Por un lado, la producción tiene un efecto inmediato sobre las reacciones tanto de compradores como de vendedores en los mercados, por lo tanto, es de esperar que las producciones tengan igualmente un efecto directo sobre las fluctuaciones de precios. Si se espera un determinado volumen de producción, la oferta y la demanda se ajustarán a estos patrones de existencias. En este sentido, si no se logran los niveles de producción estimados, traería como consecuencia una disminución de la oferta efectiva y un incremento de los precios.

Las condiciones climatológicas y meteorológicas (terremotos, huracanes, olas de frío), impactan directamente en la oferta y demanda de los commodities, mientras que los ciclos climáticos influyen en su consumo. A raíz de esto, se ven igualmente afectados sus precios. 
Por otro lado, las reservas se refieren a los niveles de stock con que se cuente de determinado activo. En correspondencia con las cantidades físicas en existencias, y la relación entre la

oferta y la demanda, el precio se moverá al alza o a la baja.

Las condiciones económicas, ya sea para la economía nacional como para la internacional, tienen un papel protagónico en el proceso de toma de decisiones de precios de los bienes. En caso de una expansión en la economía mundial, los niveles de consumo, y por tanto la demanda, se incrementan, en relación a la oferta, lo que se traduce en un aumento de los precios. $\mathrm{Si}$, en consecuencia, los productores del bien responden con un incremento desmedido en la producción, y la oferta supera la demanda, entonces los precios se deprimirán. Esta dependencia del ciclo económico es peligrosa en tanto se incrementa la volatilidad en los mercados físico y financiero.

Conocer acerca del comportamiento de la inflación resulta relevante para el análisis fundamental, pues indica, a su vez, las variaciones de los precios en una economía dada y, por tanto, la competitividad de esta economía frente al resto del mundo.

\section{Análisis técnico}

Junto al análisis fundamental, el análisis técnico es muy utilizado actualmente por los especialistas. Este parte de la premisa de que el precio de un activo en el mercado incorpora ya toda la información relevante que puede impactarlo, de modo que el estudio del comportamiento del precio puede llegar a explicar movimientos futuros del mismo. Muchos inversores combinan ambos tipos de análisis para tomar decisiones respecto a la gestión de sus operaciones en el mercado, de modo que se aprovechen las diversas ventajas que ofrece cada uno de ellos. El Análisis Técnico se basa en las operaciones estadísticas y matemáticas con las variables para detectar las tendencias y facilitar la toma de decisiones. De manera general, requiere de la utilización de gráficos para facilitar el proceso de toma de decisiones cuya confección se basa en las informaciones referidas a los precios o cotizaciones y los volúmenes de negociación del producto específico. Su interpretación se desarrolla a partir de encontrar formaciones típicas que indiquen cambios en las tendencias de los valores.

\section{Herramientas para el análisis de riesgos de mercado}

La formación del precio de los instrumentos derivados es un elemento de gran significación para la negociación en los mercados financieros. Si bien los análisis fundamental y técnico influyen de manera determinante en la toma de decisiones, la subjetividad inherente a ellos continúa siendo su principal debilidad. Para darle respuesta a este inconveniente, se utilizan herramientas basadas en ciencias exactas que añaden rigor científico, por la objetividad que imprimen a los análisis.

En este trabajo se presenta una aproximación de los precios del petróleo y aluminio con la ayuda de herramientas matemáticas, específicamente de las ecuaciones diferenciales 
estocásticas, y del software Matlab, que permitió la obtención de parámetros del modelo a, $\mu$, o que describen los precios. Luego de una búsqueda de datos se logró encontrar un modelo que describe el comportamiento en el tiempo de los precios del aluminio y el petróleo.

\section{Ecuaciones diferenciales estocásticas:}

Las aplicaciones de las ecuaciones diferenciales estocásticas (EDE) abarcan múltiples campos de las matemáticas, física, demografía y economía. A diferencia de las ecuaciones diferenciales ordinarias (EDO), las EDE tienen un término aleatorio de perturbación que hace que el conjunto solución no sea una curva suave, sino una trayectoria continua llena de altibajos con picos en todo el intervalo.

Una EDE se identifica mediante la siguiente ecuación:

$$
d S=+(S, t) d t+g(S, t) d B
$$

Donde $\mathrm{S}$ es la función que depende del tiempo (t), B es un proceso estocástico del tipo browniano, $\mathrm{y} \uparrow \mathrm{y} \mathrm{g}$, son dos funciones. (Mikosch, 2004)

En la Economía, se utilizan este tipo de ecuaciones para describir los precios de ciertas materias primas, y se les llama "Procesos de reversión a la media", un tipo de EDE con la estructura específica que se observa:

$$
d S=a(\mu-S) d t+o S d B
$$

Donde $S$ representa el precio de la materia prima, $\mu$ es la media o valor esperado, a representa la rapidez con la que $\mathrm{S}$ se aproxima a su media y o está asociado a la desviación del modelo, o sea que tan dispersos se encuentran los datos de la media. (Marin, Ecuaciones diferenciales estocásticas y casos de aplicación en finanzas, 2007)

Auxiliándose de un lenguaje de programación se pueden obtener los parámetros del modelo a, $\mu$, o que describen los precios de acuerdo a lo descrito en (Marin \& Palacio, Gaussian Estimation of One-Factor Mean Reversión, 2013). Específicamente se utilizó el software Matlab (Anexos).

\section{Comportamiento de los precios del petróleo.}

Para la realización del modelo se contó con 252 datos. Los datos fueron tomados diariamente de forma aproximada desde 2016 hasta 2017. Como se muestra en la siguiente ilustración el comportamiento real de los precios del petróleo. Los precios fueron escalados. Todos los gráficos que se presentan a continuación muestran el precio en función del tiempo (días).

Gráfico N 1. Comportamiento en la realidad de los precios del petróleo. 


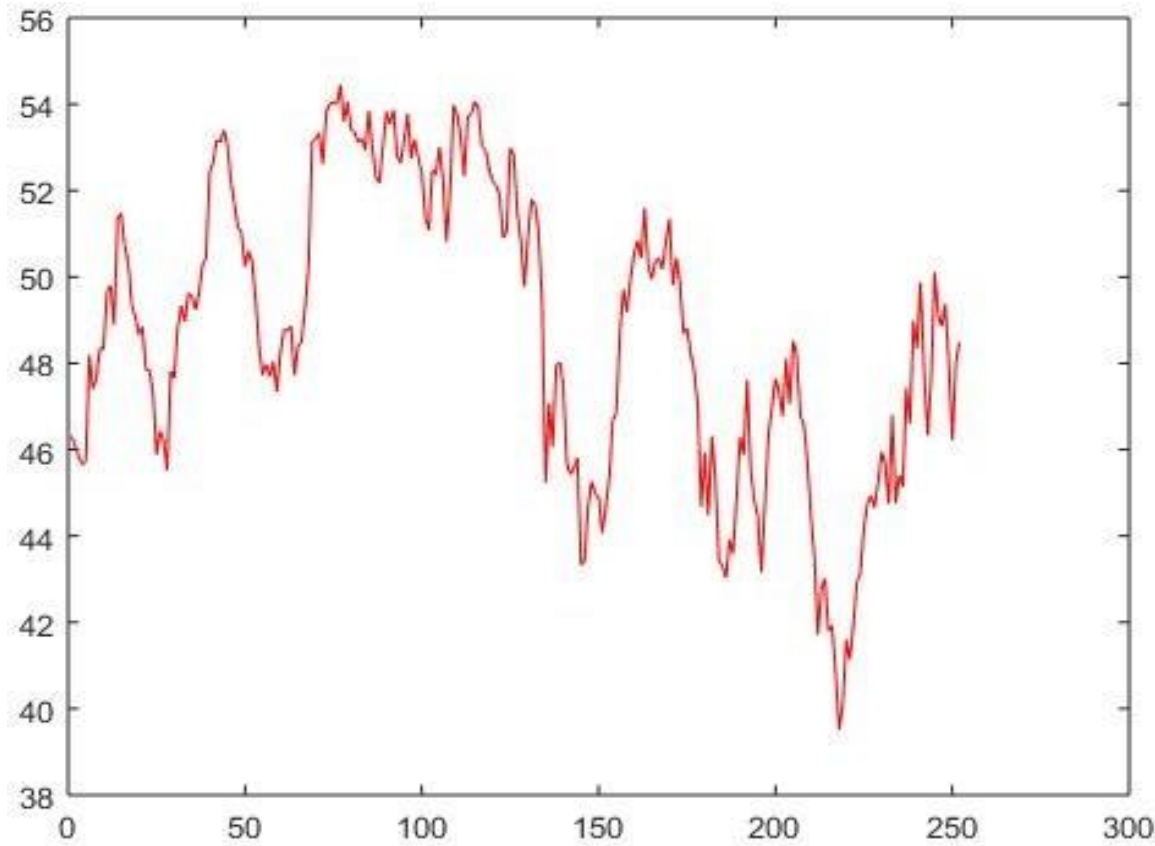

Fuente: Elaboración propia.

Utilizando el software Matlab se encontraron los parámetros necesarios para así mostrar la ecuación que describe el fenómeno. Estos parámetros se encuentran desarrollando la metodología descrita en (Marin \& Palacio, Gaussian Estimation of One-Factor Mean Reversion, 2013)

$$
\begin{aligned}
& a=11,8948 \\
& \mu=48,9130 \\
& o=0,3463
\end{aligned}
$$

Siendo la EDE dS = 11, 8948(48, $9130-\mathrm{S}) \mathrm{dt}+0,3463 \cdot \mathrm{SdB}$

Luego utilizando el mismo software ya con los parámetros de la EDE se puede observar cómo se ajusta la ecuación a la realidad:

Gráfico N 2. Ajuste de la ecuación a la realidad del modelo. Rojo precios reales, azul precios estimados. 


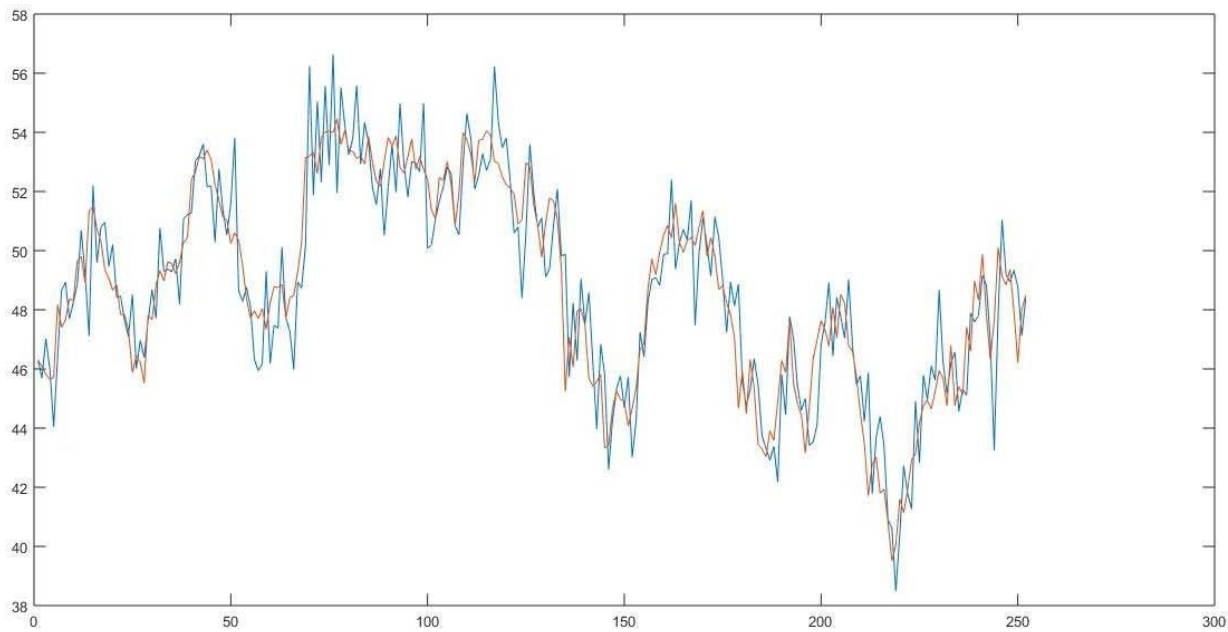

Fuente: Elaboración propia.

De la misma manera gracias al mismo software fue posible encontrar una aproximación de cómo se comportarían estos precios un año después como se muestra a continuación.

Gráfico N 3. Ajuste de la ecuación a la realidad del modelo. Rojo precios reales, azul precios estimados.

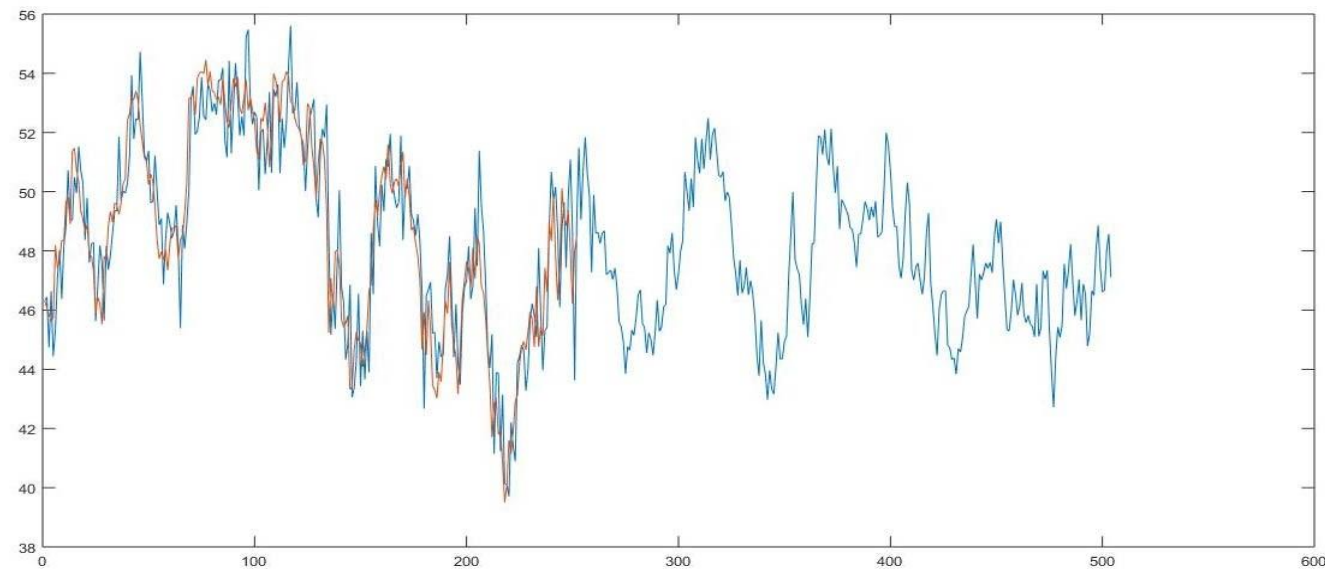

Fuente: Elaboración propia.

Como estos fenómenos son aleatorios, por lo tanto, incontrolables, la imagen anterior solo muestra una posible trayectoria de los precios. Para realizar una predicción exacta se deben hallar muchas posibles trayectorias y se obtendría un rango en el que se moverán los precios, por ejemplo, para 10 futuras trayectorias se comportaría aproximadamente como se muestra a continuación:

Gráfico N 4. Comportamiento de los precios del petróleo. 


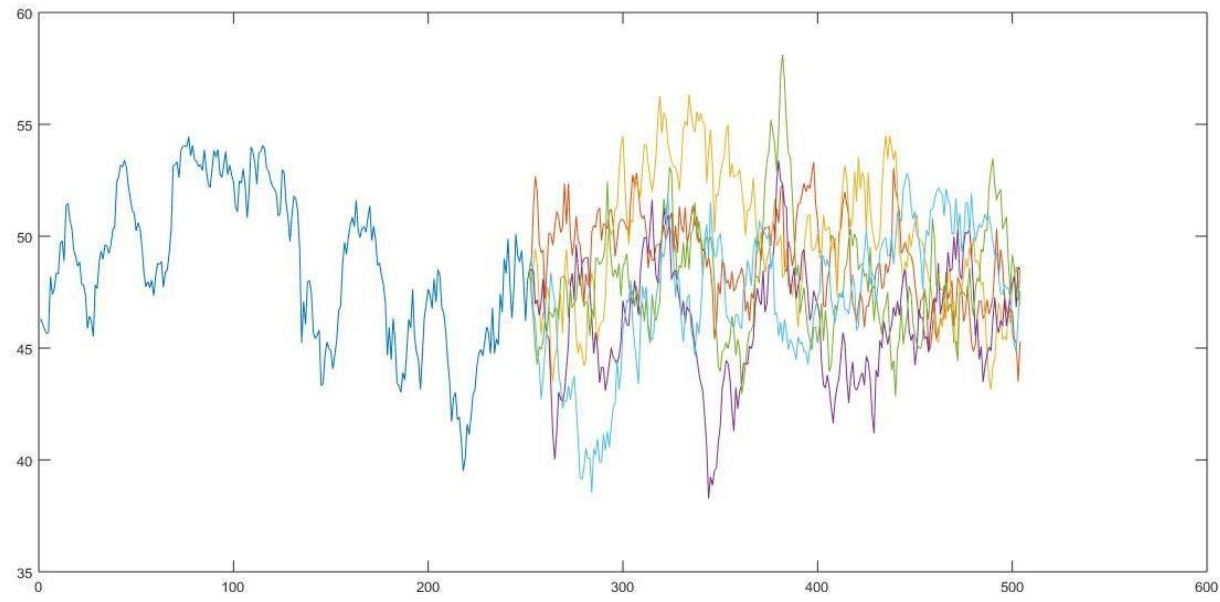

Fuente: Elaboración propia.

Para la realización del modelo se contó con 1405 datos para así lograr un mejor ajuste. Los datos fueron tomados diariamente de forma aproximada desde 2011 hasta 2017. Como se muestra en la siguiente ilustración el comportamiento real de los precios del aluminio. Los precios fueron escalados.

Gráfico N 5. Comportamiento en la realidad de los precios del aluminio

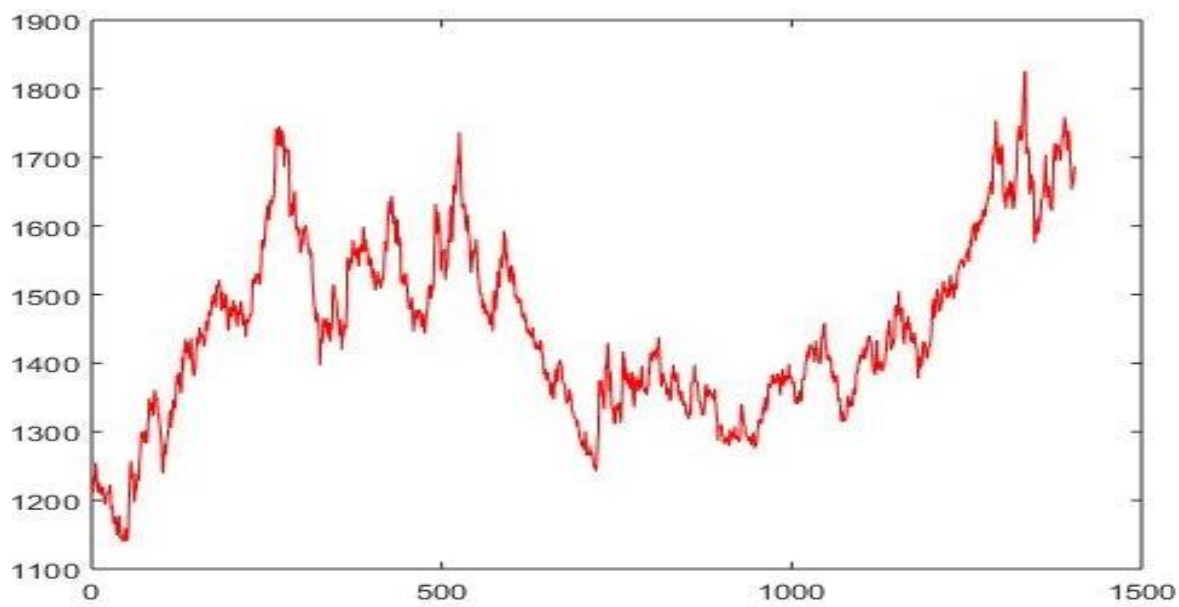

Fuente: Elaboración propia.

Utilizando el software Matlab se encontraron los parámetros necesarios para así mostrar la ecuación que describe el fenómeno.

$$
\begin{gathered}
a=1.6404 \\
\mu=1.5053 \cdot 103 \\
o=0.2027
\end{gathered}
$$


Siendo la EDE dS $=1.6404(1.5053 \cdot 103-\mathrm{S}) \mathrm{dt}+0.2027 \cdot \mathrm{SdB}$

Luego utilizando el mismo software ya con los parámetros de la EDE se puede observar cómo se ajusta la ecuación a la realidad:

Gráfico N 6. Ajuste de la ecuación a la realidad del modelo. Rojo precios reales, azul precios estimados.

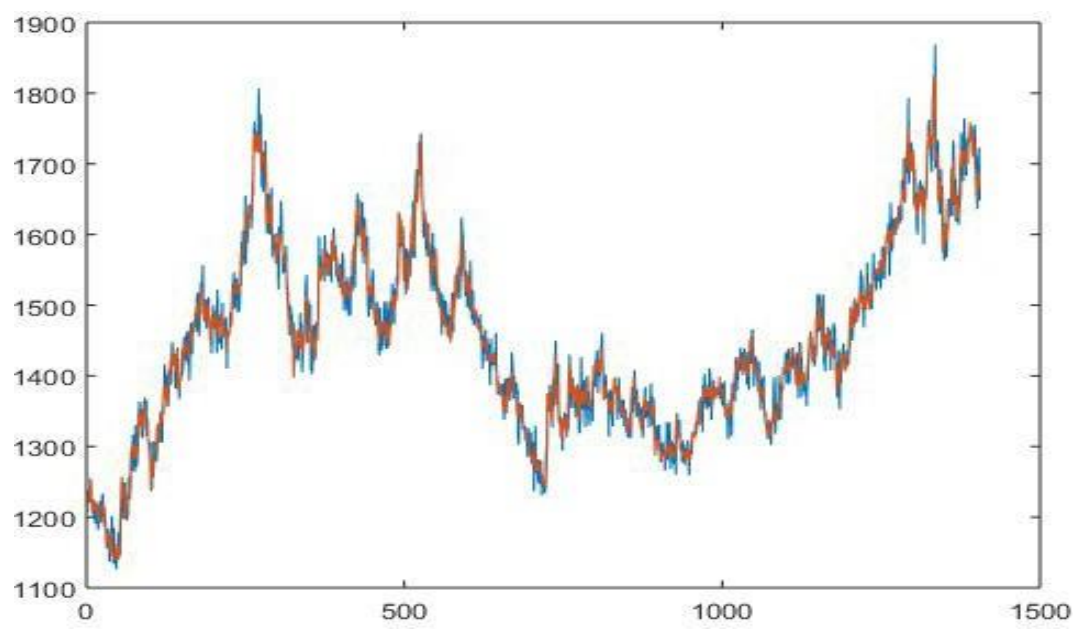

Fuente: Elaboración propia.

De la misma manera gracias al mismo software fue posible encontrar una aproximación de cómo comportarían estos precios un año después como se muestra a continuación.

Gráfico N 7. Ajuste de la ecuación a la realidad del modelo. Rojo precios reales, azul precios estimados.

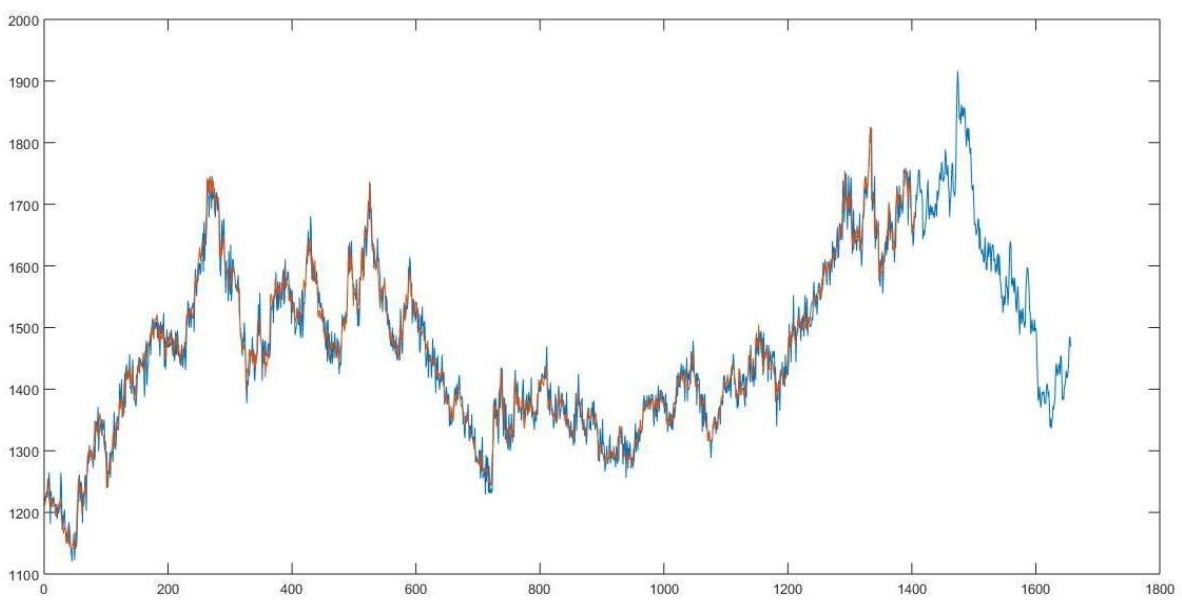

Fuente: Elaboración propia. 
Como se explicaba anteriormente, para una correcta predicción a corto plazo, se deben realizar múltiples trayectorias de los precios del aluminio. La siguiente imagen muestra 10 trayectorias. Al estar presente la incertidumbre existen varias posibilidades al estar definido el proceso en parte por variables aleatorias.

Gráfico N 8. Comportamiento de los precios del aluminio.

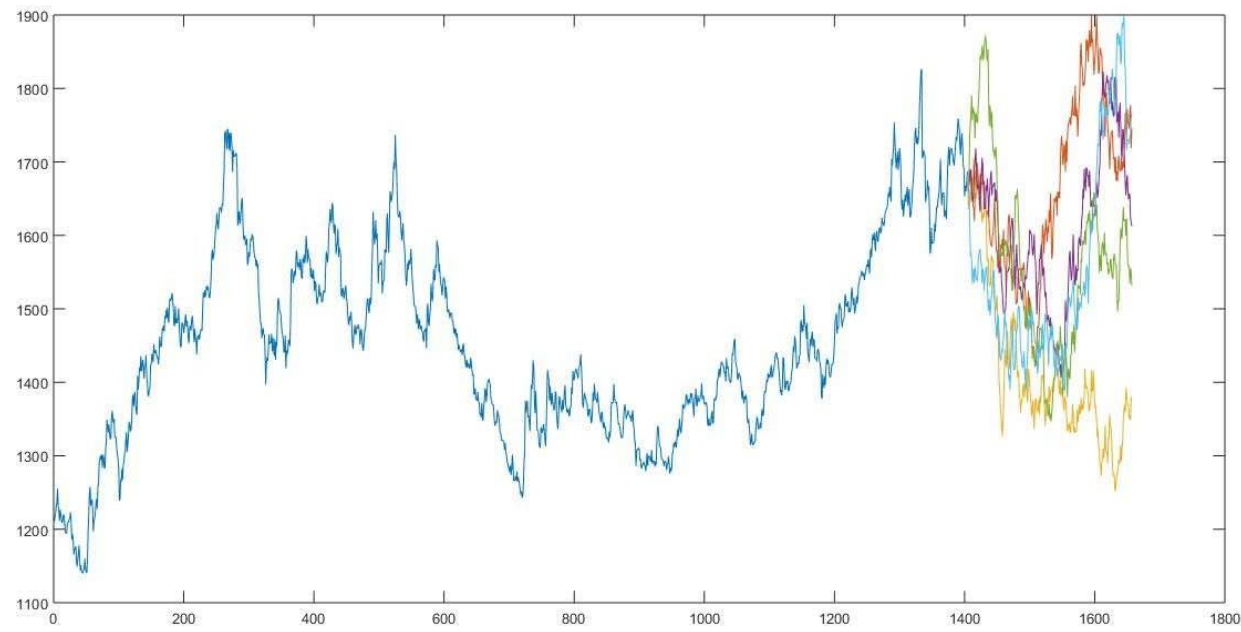

Fuente: Elaboración propia.

Demostrando de esta manera cuán provechoso puede ser esta herramienta para la toma de decisiones y anticiparse a las fluctuaciones del mercado con los mayores beneficios para la nación.

\section{Conclusiones}

- Se quisiera resaltar lo útil que ha resultado la realización del trabajo para poder entender mejor algunos aspectos de la carrera y cómo se apoya en la Matemática para la resolución de disímiles problemas y elaboración de teorías económicas.

- Desde el punto de vista económico y aplicando el herramental previamente definido, las ecuaciones diferenciales son empleadas en lo que se conoce como matemática financiera en la capitalización continua del interés; así como en el proceso de toma de decisiones en la administración de riesgo para aproximar los precios a la realidad de materias primas como petróleo y aluminio.

- El modelo que describe al petróleo es menos exacto que el del aluminio, esto fue provocado pues esa modelación se realizó con una cantidad menor de datos. Evidentemente a mayor cantidad de datos mayor exactitud tendrá el modelo, pero aun así la herramienta permitió llegar a una aproximación muy cercana a la realidad. 
- Se recomienda seguir indagando sobre tan interesante temática que sin dudas está en constante evolución y es un vasto campo de investigación tanto para economistas como para matemáticos.

\section{Referencias bibliográficas.}

Marin, F. H. (2007). Ecuaciones diferenciales estocasticas y casos de aplicacion en finanzas.

Tesis de Maestria.

Marin, F. H., \& Palacio, J. S. (2013). Gaussian Estimation of One-Factor Mean Reversion.

Hindawi Publishing Corporation.

Mikosch, T. (2004). Elementary stochastic calculus with. World Scientific. Shampine, L. F. (s.f.). Solving ODEs with MATLAB.

Zill, D. G. (1997). Differential Equations with Modeling Applications.

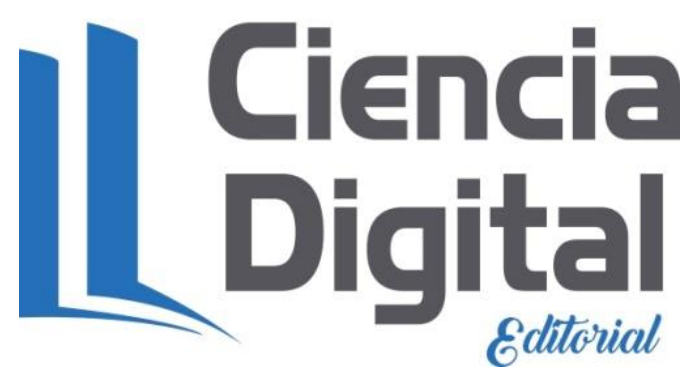


Para citar el artículo indexado.

Panelas Ramos, F., Rivera Rodríguez, C., \& Santos Fernández, K. (2018). Predicción de precios del petróleo y el aluminio usando ecuaciones diferenciales estocásticas. ConcienciaDigital, 1(4), 45-58. https://doi.org/10.33262/concienciadigital.v1i4.906

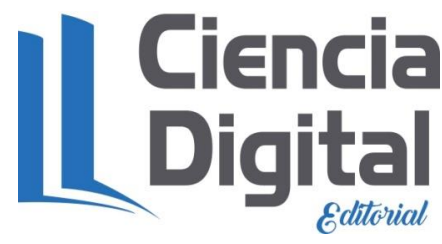

El artículo que se publica es de exclusiva responsabilidad de los autores y no necesariamente reflejan el pensamiento de la Revista Conciencia Digital.

El articulo queda en propiedad de la revista y, por tanto, su publicación parcial y/o total en otro medio tiene que ser autorizado por el director de la Revista Conciencia Digital.

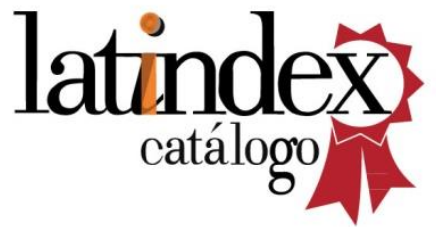

